\title{
Clarifying the Role of Neural Networks in Complex Hallucinatory Phenomena
}

\author{
Claire 0’Callaghan, ${ }^{1}$ Alana J. Muller, ${ }^{2}$ and ${ }^{\circ}$ James M. Shine ${ }^{2}$ \\ ${ }^{1}$ Neuroscience Research Australia and School of Medical Sciences, University of New South Wales, Sydney, NSW 2052, Australia, and ${ }^{2}$ Brain and Mind \\ Research Institute, University of Sydney, Sydney, NSW 2006, Australia \\ Review of Mégevand et al.
}

Visual hallucinations and related phenomena, such as déjà vu, are reminders that conscious perception does not always accurately reflect external reality. We might be startled by a garden hose that at first glance looks like a snake, or feel an eerie sense of familiarity in new surroundings. For the most part these are transitory and intriguing perceptual glitches, yet in many neuropsychiatric disorders they are recurrent and troubling symptoms. Currently there is no unified brain-behavior framework to conceptualize these phenomena. The work by Mégevand and colleagues (2014), which uses intracranial EEG to discern the neural correlates of topographic visual hallucinations and déjà vu in the parahippocampal place area, is timely and informative, hinting at a mechanism for these phenomena involving interactions between large-scale neural networks.

Visual hallucinations range from the simple to the complex. Simple hallucinations are typically abstract shapes or patterns, and they can be induced via electrical brain stimulation to the primary and secondary visual cortices (Selimbeyo-

Received June 13, 2014; revised July 18, 2014; accepted July 18, 2014.

C. 0 . is supported by an Alzheimer's Australia Dementia Research Foundation PhD scholarship. A.J.M. is supported by an Australian Postgraduate Research Award. J.M.S. is supported by Supported by a Michael J. Fox Project Grant.

Correspondence should be addressed to Dr James M. Shine, 94 Mallet Street, Camperdown, 2050 NSW, Australia. E-mail: mac.shine@sydney.edu.au.

DOI:10.1523/JNEUROSCI.2429-14.2014

Copyright $\odot 2014$ the authors $\quad 0270-6474 / 14 / 3411865-03 \$ 15.00 / 0$ glu and Parvizi, 2010). In contrast, complex hallucinations comprise recognizable objects, such as people, animals, or topographical scenery, which are often imbued with personal meaning. The elaborate quality of complex hallucinations suggests involvement of regions outside the primary visual areas, and indeed, they are widely reported during electrical stimulation of medial temporal lobe sites, including the hippocampus and amygdala, fusiform and parahippocampal gyri, and entorhinal and perirhinal cortices (Selimbeyoglu and Parvizi, 2010). Similarly, déjà experiences exist on a continuum, with déjà vu representing the attenuated form of familiarity in a novel context, and déjà vécu the more encompassing experience of having previously lived through a certain situation (Illman et al., 2012). Déjà phenomena are also strongly associated with medial temporal lobe disturbances, most commonly manifesting experimentally with electrical stimulation of the amygdala, hippocampus, and parahippocampal cortex (Vignal et al., 2007).

Despite the broad implication of the parahippocampal cortex in complex hallucinatory phenomena, its posterior region, the parahippocampal place area (PPA), specialized for processing visual scenes, had not previously been linked to topographic hallucinations. The PPA is historically defined as the posterior subregion of the parahippocampal cortex, although more recent evidence confirms peak activation for scene-selective pro- cessing can extend to the lateral edge of the collateral sulcus, adjacent to the medial fusiform gyrus (Nasr et al., 2011). Mégevand et al. (2014) demonstrate a direct link between the PPA and topographic hallucinations, thereby providing evidence of a role for PPA neuronal activity in conscious scene perception. Importantly, their work further confirms the significance of the parahippocampal region in complex visual hallucinations and déjà vu. Framing these results within the context of broader neural networks that interact with the PPA may offer insight into the mechanisms that underpin these complex phenomenological experiences in clinical disorders.

In an individual undergoing presurgical evaluation for drug-resistant epilepsy, Mégevand and colleagues (2014) electrically stimulated the PPA. Before electrode implantation, functional MRI data was collected during presentation of pictures of buildings, faces, man-made objects, and geometric patterns. By contrasting peak BOLD response for buildings versus responses for other picture categories, a scene-selective region corresponding to the PPA was identified in the collateral sulcus and medial fusiform gyrus of the basal temporal lobe. Selectivity of this region was further confirmed with intracranial EEG recordings, by analyzing changes in broadband high-power gamma band $(40-160 \mathrm{~Hz})$ in response to presentation of houses versus faces and other objects. Those sites confirmed as scene-selective 
via converging evidence from the functional MRI and intracranial EEG were then targeted for direct stimulation.

Bipolar electrical stimulation was delivered via $200-\mu$ s, square-wave biphasic pulses at $50 \mathrm{~Hz}$ for $\sim 2 \mathrm{~s}$ at 11 sites in the predefined scene-selective region. An additional 38 electrode pairs were stimulated in the temporal and frontal lobes, although none induced abnormal percepts. Before each stimulation trial, the subject was asked to report whether they saw, heard, or felt anything. Two stimulation sites in the fusiform gyrus elicited topographic hallucinations: one was a detailed scene of a train station in the patient's neighborhood (medial fusiform gyrus), the other an unrecognized scene of a closet and a staircase (lateral fusiform gyrus). The patient described passively viewing the scenes as if they were presented in front of him, without the feeling of actually being there. In contrast, stimulation of a slightly more lateral region in the inferior temporal gyrus resulted in distorted perception of the investigators' faces, and also a complex visual phenomenon involving face-body distortion with a topographic context (i.e., investigators "looked Italian", with aprons, as if they worked in a pizza shop). Interestingly, stimulation to the rostrolateral aspect of the fusiform gyrus induced a combination of strong déjà vu and a complex topographical hallucination of a familiar street scene. Stimulation of the medial aspect of this rostral region elicited déjà vu without an accompanying visual percept.

Together, the findings suggest that stimulation of peak scene-selective regions in the PPA result in complex topographical hallucinations, while stimulation of outer regions results in an admixture of topographic and other perceptual disturbances, including déjà vu. This provides direct causal evidence that localized neuronal activity in the human PPA subtends perception of visual scenes, with previous correlational evidence having been garnered from functional MRI studies or indirectly from lesion studies.

Emergence of complex hallucinatory phenomena from PPA stimulation is consistent with its anatomical positioning and with the hypothesized role for the parahippocampal cortex as a site for integration and processing of contextual associations (Aminoff et al., 2013). The PPA, and more broadly the parahippocampal cortex, has dense reciprocal connections within the medial temporal lobe (i.e., temporal pole and perirhinal cortex); it provides major input to the entorhinal

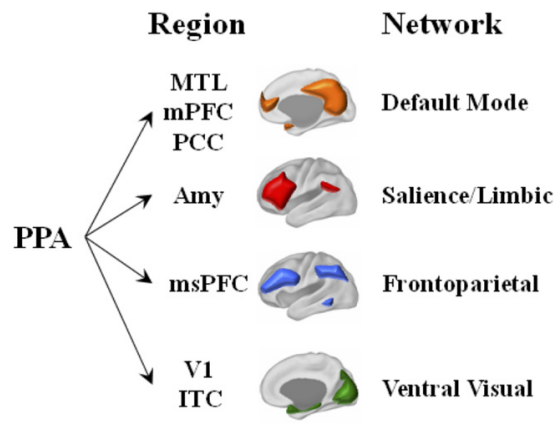

Figure 1. The PPA and its broader neural network connections. Key regions that show anatomical and functional connections with the PPA and the broader neural networks encompassing these key regions, with crucial roles in selfreferential processing and memory (default mode network); emotional processing (salience and limbic networks); cognitive control (frontoparietal network); and higher-order visual processing (ventral visual stream). PPA, Parahippocampal place area; MTL, medial temporal lobe; $\mathrm{mPFC}$, medial prefrontal cortex; PCC, posterior cingulate cortex; Amy, amygdala; msPFC, medial superior prefrontal cortex; ITC, inferotemporal cortex.

cortex, which feeds directly into the hippocampus; and it also has direct connections to the hippocampus and the amygdala. This places the PPA at the interface of higher-order visual processing (i.e., the ventral visual pathway that spans the inferotemporal cortex), self-referential processing and memory (i.e., the brain's default network, including the hippocampal and parahippocampal regions), and emotion (i.e., limbic and salience networks, including the amygdala) (Fig. 1). Abnormal activity in this integration region could conceivably produce the combination of visual, autobiographical, and emotive qualities that characterize complex hallucinations and déjà experiences.

Although Mégevand et al. (2014) reveal a direct role for PPA activity in complex hallucinations and déjà vu, such localized activity does not explain the entire spectrum of hallucinatory experiences. For instance, hallucinatory phenomena are known to occur in response to neural stimulation in extra-temporal regions and in clinical disorders characterized by extra-temporal lobe dysfunction. For example, prefrontal stimulation sites have evoked complex visual hallucinations (Blanke et al., 2000) and propagation of stimulation current to frontotemporal regions subserving autobiographical memory is thought to underpin a more encompassing experience of déjà vécu combined with visual hallucinations (Illman et al., 2012). In line with this, Herbet et al. (2014) provided evidence that direct stimulation to white matter tracts connecting the posterior cingulate region and medial prefrontal cortex-core midline structures in the default network-induced vivid imagery and the feeling of being in another place (i.e., the patient described being on the beach, as if in a dream).

A role for extra-temporal regions in clinical disorders has been indicated in Charles Bonnet syndrome, in which functional MRI has linked complex visual hallucinations to aberrant activity in the secondary visual cortex (Ffytche et al., 1998); Parkinson's disease, in which dysfunction in the dorsal frontoparietal attention system is documented during complex visual hallucinations (Shine et al., 2014); and in schizophrenia with visual hallucinations, which is associated with hyperconnectivity between the hippocampus and the occipital cortex, medial prefrontal, and striatal regions (Amad et al., 2014).

Considering the direct link between the PPA and hallucinations that Mégevand et al. (2014)demonstrate, might dysfunction in prefrontal and occipital sites, or their afferent projections, be a source of aberrant neural activity in the $\mathrm{PPA}$, giving rise to complex hallucinatory phenomena in clinical disorders? As illustrated by both functional and anatomical connectivity studies, the PPA lies at the nexus of large-scale attentional and perceptual networks (Fig. 1). For example, functional connectivity studies demonstrate the posterior PPA is strongly connected to early visual regions in the occipital cortex, while the anterior PPA is specifically connected to a constellation of regions that overlaps with the default network, and indirectly connected with medial superior frontal regions (Baldassano et al., 2013). Modulation of the PPA by these distal regions has been described. For example, dependent on the information processing required for incoming visual information, the frontoparietal network has been shown to enhance PPA activity, and the default network to suppress PPA activity (Chadick and Gazzaley, 2011). This places the PPA in an ideal position to be influenced by bottom-up activity from the visual cortex, and also top-down modulation from large-scale attentional and perceptual networks.

Given that highly specific regions in the PPA evoke complex hallucinatory phenomena, consideration of how dysfunction in large-scale neural networks and distal brain regions impinges upon the PPA will certainly help to clarify the pathophysiological mechanism of hallucinations across clinical disorders with 
heterogeneous pathology. A further intriguing consideration is that while this circumscribed aberrant activity in the PPA produced a feeling of familiarity and of passively viewing topographic hallucinations, expansive aberrant activity in default network regions (Illman et al., 2012; Herbet et al., 2014) can induce a more encompassing déjà vécu and the feeling of being embedded in the hallucinated topography. This comparison may explain why chronic visual hallucinations in schizophrenia and Parkinson's disease, which are associated with more diffuse pathology, can often become increasingly vivid and difficult to separate from reality. Considering the findings of Mégevand et al. (2014) in such a framework highlights that pathological complex hallucinatory experiences are best considered a network phenomenon.

\section{References}

Amad A, Cachia A, Gorwood P, Pins D, Delmaire C, Rolland B, Mondino M, Thomas P, Jardri R (2014) The multimodal connectivity of the hippocampal complex in auditory and visual hallucinations. Mol Psychiatry 19:184-191. CrossRef Medline

Aminoff EM, Kveraga K, Bar M (2013) The role of the parahippocampal cortex in cognition. Trends Cogn Sci 17:379-390. CrossRef Medline

Baldassano C, Beck DM, Fei-Fei L (2013) Differential connectivity within the parahippocampal place area. Neuroimage 75:228-237. CrossRef Medline

Blanke O, Landis T, Seeck M (2000) Electrical cortical stimulation of the human prefrontal cortex evokes complex visual hallucinations. Epilepsy Behav 1:356-361. CrossRef Medline

Chadick JZ, Gazzaley A (2011) Differential coupling of visual cortex with default or frontalparietal network based on goals. Nat Neurosci 14:830-832. CrossRef Medline

Ffytche DH, Howard RJ, Brammer MJ, David A, Woodruff P, Williams S (1998) The anatomy of conscious vision: an fMRI study of visual hallucinations. Nat Neurosci 1:738-742. CrossRef Medline

Herbet G, Lafargue G, de Champfleur NM, Moritz-Gasser S, le Bars E, Bonnetblanc F, Duffau H (2014) Disrupting posterior cingulate connectivity disconnects consciousness from the external environment. Neuropsychologia 56:239-244. CrossRef Medline

Illman NA, Butler CR, Souchay C, Moulin CJ
(2012) Déjà experiences in temporal lobe epilepsy. Epilepsy Res Treat 2012:539567. CrossRef Medline

Mégevand P, Groppe DM, Goldfinger MS, Hwang ST, Kingsley PB, Davidesco I, Mehta AD (2014) Seeing scenes: topographic visual hallucinations evoked by direct electrical stimulation of the parahippocampal place area. J Neurosci 34:5399-5405. CrossRef Medline

Nasr S, Liu N, Devaney KJ, Yue X, Rajimehr R, Ungerleider LG, Tootell RB (2011) Sceneselective cortical regions in human and nonhuman primates. J Neurosci 31:13771-13785. CrossRef Medline

Selimbeyoglu A, Parvizi J (2010) Electrical stimulation of the human brain: perceptual and behavioral phenomena reported in the old and new literature. Front Hum Neurosci 4:46. CrossRef Medline

Shine JM, Halliday GM, Gilat M, Matar E, Bolitho SJ, Carlos M, Naismith SL, Lewis SJ (2014) The role of dysfunctional attentional control networks in visual misperceptions in Parkinson's disease. Hum Brain Mapp 35:22062219. CrossRef Medline

Vignal JP, Maillard L, McGonigal A, Chauvel P (2007) The dreamy state: hallucinations of autobiographic memory evoked by temporal lobe stimulations and seizures. Brain 130: 88-99. CrossRef Medline 